\title{
The Effect of Elastic Properties on Indentation Test for Multi-layered Materials
}

\author{
Kotaro Miura $^{1 *}$, Makoto Sakamoto ${ }^{2}$, and Yuji Tanabe ${ }^{3}$ \\ ${ }^{1}$ Department of Systems Design Engineering, Seikei University, 3-3-1 Kichijoji-kitamachi, Musashino-shi, Tokyo, Japan \\ 2 Department of Health Sciences, Niigata University School of Medicine, 2-746 Asahimachi, Niigata, Japan \\ ${ }^{3}$ Graduate School of Science and Technology, Niigata University, 2-8050, Ikarashi, Niigata, Japan \\ *k_miura@st.seikei.ac.jp
}

Introduction. Indentation tests have been widely used to mechanical properties of materials such as industrial products and biological tissues. The above test is simple and flexible since it is not necessary to process materials into standard shape of specimens. The technology of coating of materials such as functionally graded materials (FGMs) to improve in the wear resistance of surface has been developing in recent years. FGMs materials can be considered as a limiting condition of multi-layered structures. In the field of biology, many of biological tissues can be regarded as multi-layered structures. Therefore, it is important to establish the foundation of theory of indentation tests for multilayered materials. In this study, the axisymmetric contact problem of multi-layer material bonded on elastic semi-infinite substrate indented by flat-ended cylindrical and spherical rigid punch was considered. Numerical results of axial load of rigid punch when two specific case of mechanical properties of multi-layered materials were presented.

Methods of Analysis. We consider the problem where a composite material consisting of arbitrary number of elastic layers perfectly bonded to an elastic semi-infinite substrate is indented by a rigid flat-ended cylindrical indenter or a spherical indenter, as shown in Fig. 1. The contact problem which is boundary-value problem usually reduced to the second kind of Fredholm integral equations. In this study, dual integral equations obtained from governing equations were reduced to an infinite system of simultaneous equations by expressing a normal contact stress at the surface elastic layer as an appropriate series with Tchebycheff orthogonal polynomial $^{(1)}$. Furthermore, the information of each elastic layers can be sorted by using transfer matrix method $^{(2)}$ and multi-layered problem can be treated the same as the contact problem for one layer bonded to semi-infinite substrate.

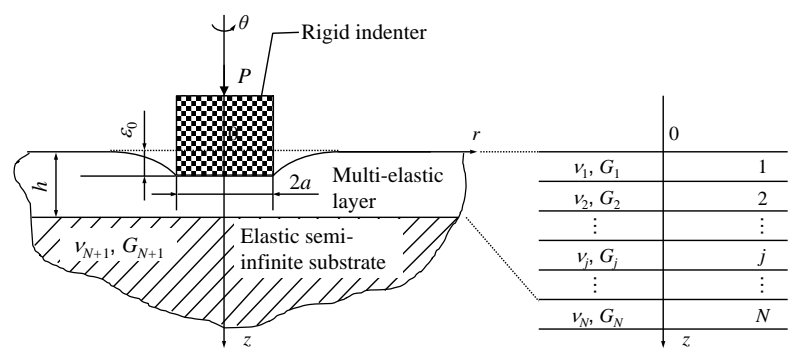

Fig. 1 Scheme of indentation of multi-layered material bonded on semi-infinite substrate.
Numerical results and Discussions. Figure 2 shows the numerical results of axial load of flat-ended cylindrical and spherical rigid punch for one-layered and multi-layered materials. The multi-layered material has the same ratio of shear modulus of surface to substrate as that of one-layered material, $G_{1}$ (surface layer) $/ G_{N+1}$ (substrate) $=0.2$ and 5.0. $P_{1}$ indicates the axial load needed to indentation for half-space with shear modulus of surface layer. The variation of shear modulus inside multi-layered material was assumed to be linearity and Poisson's ratio of each layer and substrate were fixed as constant values, 0.3. As shown in Fig. 2, the axial load of multi-layer is higher than one-layer when $G_{1} / G_{N+1}=$ 0.2 , which represents soft-coating system, whereas axial load of multi-layer is lower than that of one-layer when $G_{1} / G_{N+1}=5.0$, which represent hard-coating system. Therefore, hard-coating and multi-layer system has good effective in developing wear resistance of materials. Furthermore, the difference of axial load between multi- and one-layer when total thickness ratio $h / a$ is become thinner or thicker because the effect of elastic substrate or surface layer on axial load become dominant, respectively.

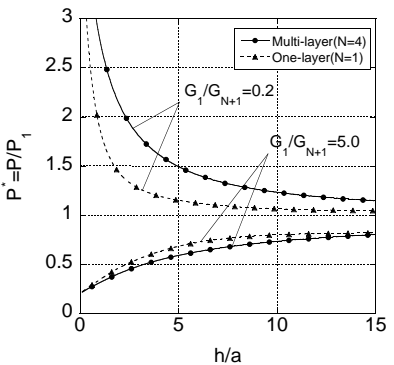

(a)

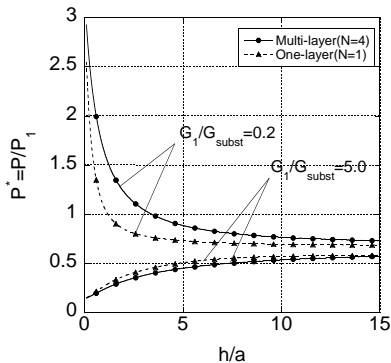

(b)
Fig. 2 Normalized axial load $P^{*}=P / P_{1}$ for multi- and one-layered material for below (a) the flat-ended cylindrical and (b) the spherical indenter.

Conclusion. The contact problem where a composite material consisting of arbitrary number of elastic layers perfectly bonded to an elastic semi-infinite substrate is indented by a rigid flat-ended cylindrical indenter or a spherical indenter was solved analyticaly.

\section{References.}

(1) Miura, K., Sakamoto, M., Kobayashi, K., Pramudita, J. A. and Tanabe, Y., Theor. Appl. Mech. Japan., 64, (2018), 81-101.

(2) Liu, T. J. and Xing, Y. M., Int. J. Mech. Sci., 81, (2014), 158-164. 